\title{
An Analysis of the Optimal Control Modification Method Applied to Flutter Suppression
}

\author{
Michael Drew* \\ Stinger Ghaffarian Technologies, Inc., Moffett Field, CA 94035 \\ Nhan T. Nguyen ${ }^{\dagger}$ \\ NASA Ames Research Center, Moffett Field, CA 94035 \\ Kelley E. Hashemi ${ }^{\ddagger}$ \\ Universities Space Research Association, Moffett Field, CA 94035 \\ Eric Ting ${ }^{\S}$ \\ NASA Ames Research Center, Moffett Field, CA 94035 \\ Daniel Chaparro \\ MORI Associates, Moffett Field, CA 94035
}

\begin{abstract}
Unlike standard Model Reference Adaptive Control (MRAC), Optimal Control Modification (OCM) has been shown to be a promising MRAC modification with robustness and analytical properties not present in other adaptive control methods. This paper presents an analysis of the OCM method, and how the asymptotic property of OCM is useful for analyzing and tuning the controller. We begin with a Lyapunov stability proof of an OCM controller having two adaptive gain terms, then the less conservative and easily analyzed OCM asymptotic property is presented. Two numerical examples are used to show how this property can accurately predict steady state stability and quantitative robustness in the presence of time delay, and relative to linear plant perturbations, and nominal Loop Transfer Recovery (LTR) tuning. The asymptotic property of the OCM controller is then used as an aid in tuning the controller applied to a large scale aeroservoelastic longitudinal aircraft model for flutter suppression. Control with OCM adaptive augmentation is shown to improve performance over that of the nominal non-adaptive controller when significant disparities exist between the controller/observer model and the true plant model.
\end{abstract}

\section{Introduction}

$\mathrm{C}^{\mathrm{OR}}$ several decades, adaptive control has been an active area of research with the promise of increasing $\mathrm{F}$ the performance of systems with unmodeled dynamics and disturbances. In particular, model reference adaptive control (MRAC), which attempts to directly modify gains in order to force the plant to match the performance of an ideal model, has been the focus of research for many years. Several modifications have been proposed to add robustness and performance attributes not present in basic MRAC. Although large adaptive learning rate is desirable to quickly reduce tracking error, it is well known that instability and extreme transients can result produce high frequencies exciting unmodeled plant dynamics. ${ }^{1}$ Proposed techniques that address this issue include $L_{1}$ adaptive control, ${ }^{2}$ adaptive loop transfer recovery, ${ }^{3}$ and Kalman filter adaptive control ${ }^{4}$ among others. Popular modifications of MRAC control that increase robustness by damping the adaption rate include $\sigma$-modification, ${ }^{5} e$-modification, ${ }^{6}$ and optimal control modification (OCM). ${ }^{7,8}$ OCM has measurable robustness properties, and has been shown to be capable of controlling

\footnotetext{
*Research Engineer, Stinger Ghaffarian Technologies, Inc., NASA Ames Research Center, michael.c.drew@nasa.gov

${ }^{\dagger}$ Research Scientist, Intelligent Systems Division, nhan.t.nguyen@nasa.gov, AIAA Associate Fellow

$\ddagger$ NASA Post-Doctoral Fellow, Intelligent Systems Division, kelley.e.hashemi@nasa.gov

§Research Engineer, Intelligent Systems Division, eric.b.ting@nasa.gov

『Research Engineer, Intelligent Systems Division, daniel.chaparro@nasa.gov
} 
non-minimum phase plants using a non-minimum phase reference model. ${ }^{9}$ It has also been successfully implemented and validated on a NASA F/A-18 aircraft. ${ }^{10}$

The OCM MRAC controller features a robustness tuning parameter $\nu$ in addition to the learning rate gain $\Gamma$. When $\nu=0$, the resulting controller is the basic MRAC. As $\nu$ increases, robustness increases, but at the cost of tracking performance. Instead of error asymptotically approaching zero, it is bounded. Bounds for the $\nu$ parameter that result in a stable adaptive controller can be found via Lyapunov analysis, or from the resulting asymptotic closed loop system matrix found by letting the adaptive gain $\Gamma \rightarrow \infty .{ }^{9}$ Both provide conservative bounds for $\nu$, with the Lyapunov bounds generally being significantly more conservative.

This paper presents an analysis of these concepts, initially with two toy problems, then, within the context of adaptive flutter suppression control applied to an aircraft aeroservoelastic (ASE) generic transport model (GTM). The effect that signal delay and linear plant perturbations and/or disparities between the controller model and the true plant model have on the stable bounds of $\nu$ are explored. Since adaptive control is often applied as an augmentation to a conventional baseline controller, an analysis of these bounds relative to the properties of the baseline controller are also investigated. In particular, the effect that Loop Transfer Recovery (LTR) has on the OCM stability regions is presented.

The paper is arranged as follows: Section II begins with a brief description of OCM MRAC adaptive control. Section III presents two small models that demonstrate some of the attractive qualities of OCM control. Section IV introduces the GTM model and control architecture that is implemented in a large scale model with results shown in Section V. Section VI summarizes these concepts and results.

\section{Background}

As with any control system, the notion of the plant held by the controller and observer may differ significantly from the actual plant. Here, we denote this difference using the subscript $c$ for the controller and $p$ for the plant. Thus, a disparity exists between the controller and plant models such that $\Delta A=A_{p}-A_{c}$, and so forth for $\Delta B, \Delta C$, and $\Delta D$. This disparity always exists to some degree because of controller/observer model reduction, modeling inaccuracies, or parameter drift. For simulation and analysis purposes, it is assumed that both the controller and plant models contain identical inputs and outputs, however, it is not necessary that both contain the same number of states, specifically $n_{c} \leq n_{p}$. Thus, given a plant state space realization

$$
\begin{aligned}
& \dot{x}=A_{p} x+B_{p} u \\
& y=C_{p} x+D_{p} u,
\end{aligned}
$$

the controller's notion of this plant is represented similarly but with state space matrices $\left(A_{c}, B_{c}, C_{c}, D_{c}\right)$. From this, a state observer is constructed using the optimal Kalman filter estimation method according to

$$
\begin{aligned}
& \dot{\hat{x}}=A_{c} \hat{x}+B_{c} u+L(y-\hat{y}) \\
& \hat{y}=C_{c} \hat{x}+D_{c} u .
\end{aligned}
$$

The optimal estimated state feedback LQG control is

$$
u_{n o m}=K_{l q r} \hat{x}
$$

where $K_{l q r}$ is the standard optimal state feedback LQR solution. Note that both $K_{l q r}$ and $L$ are computed based on the controller's notion of the plant. That is, $\left(A_{c}, B_{c}\right) \Rightarrow K_{l q r}$, and $\left(A_{c}, C_{c}\right) \Rightarrow L$.

It is desired to design a controller that augments Eq. (3) with an adaptive term so that the observer attempts to track a reference model. The reference model is defined according to

$$
A_{\text {ref }}=A_{c}+B_{c} K_{l q r}
$$

with $A_{r e f}$ being Hurwitz. Defining $e=x_{r e f}-\hat{x}$ as the state tracking error between the reference model states and the estimated states, the augmented control law becomes

$$
u=\underbrace{K_{l q r} \hat{x}}_{u_{\text {nom }}}+\underbrace{K_{x}(t) \hat{x}+K_{y}(t)(y-\hat{y})}_{u_{a d}}
$$


where $u_{\text {nom }}$ denotes the nominal non-adaptive control of Eq. (3), and $u_{a d}$ denotes the adaptive augmentation. $K_{x}(t)$ and $K_{y}(t)$ adapt according to optimal control modification laws ${ }^{7,8}$

$$
\begin{aligned}
& \dot{K}_{x}=B_{c}^{T}\left(P e+\nu_{x} A_{r e f}^{-T} P B_{c} K_{x} \hat{x}\right) \hat{x}^{T} \Gamma_{x} \\
& \dot{K}_{y}=B_{c}^{T}\left(P e+\nu_{y} A_{r e f}^{-T} P B_{c} K_{y}(y-\hat{y})\right)(y-\hat{y})^{T} \Gamma_{y} .
\end{aligned}
$$

Here, $\Gamma_{x}=\Gamma_{x}^{T}>0$ and $\Gamma_{y}=\Gamma_{y}^{T}>0$ are the adaptive gains. $\nu_{x}>0$ and $\nu_{y}>0$ tune the effect of the augmented modification. $P=P^{T}$ is the solution to the standard Lyapunov equation based on the reference model $P A_{\text {ref }}+A_{r e f}^{T} P+Q=0$ with $Q=Q^{T}>0$. Note that initial conditions for both adaptive laws are $K_{x}(0)=K_{y}(0)=0$. Tuning the OCM adaptive controller involves finding suitable values of $\Gamma_{x}$ and $\Gamma_{y}$ to produce fast but realistic adaptation, along with values of $\nu_{x}$ and $\nu_{y}$ that are large enough to provide robustness, but low enough to maintain acceptable tracking performance.

The following subsections present two methods for finding stabilizing limits of $\nu_{x}$ and $\nu_{y}$ based on the above control law.

\section{A. Lyapunov Stability Analysis}

Similar to the analysis in Ref. 11, a Lyapunov function can be chosen as

$$
V\left(e, e_{p}, \tilde{K}_{x}, \tilde{K}_{y}\right)=e^{T} P e+e_{p}^{T} W e_{p}+\operatorname{trace}\left(\tilde{K}_{x} \Gamma_{x}^{-1} \tilde{K}_{x}^{T}\right)+\operatorname{trace}\left(\tilde{K}_{y} \Gamma_{y}^{-1} \tilde{K}_{y}^{T}\right)
$$

where $e=x_{r e f}-\hat{x}, e_{p}=x-\hat{x}, \tilde{K}_{x}=K_{x}(t)-K_{x}^{*}$, and $\tilde{K}_{y}=K_{y}(t)-K_{y}^{*} . K_{x}^{*}$ and $K_{y}^{*}$ represent the ideal gains determined from the model matching conditions between the controller and the observer. $W$ is the solution to the observer's Lyapunov equation $W\left(A_{c}-L C_{c}\right)+\left(A_{c}-L C_{c}\right)^{T} W+R=0$.

Evaluating $\dot{V}\left(e, e_{p}, \tilde{K}_{x}, \tilde{K}_{y}\right)$ gives

$$
\begin{aligned}
& \dot{V}\left(e, e_{p}, \tilde{K}_{x}, \tilde{K}_{y}\right)=-e^{T} Q e-2 e^{T} P B_{c} \tilde{K}_{x} \hat{x}-2 e^{T} P B_{c} \tilde{K}_{y} \hat{x} \\
& \quad-e_{p}^{T} R e_{p}+2 e_{p}^{T} W \Delta A\left(e_{p}+\hat{x}\right)+2 e_{p}^{T} W \Delta B\left(K_{l q r} \hat{x}+K_{x} \hat{x}+K_{y} C_{c} e_{p}\right) \\
& \quad+2 \nu_{x} \hat{x}^{T} K_{x}^{T} B_{c}^{T} P A_{r e f}^{-1} B_{c} \tilde{K}_{x} \hat{x}+2 e^{T} P B_{c} \tilde{K}_{x} \hat{x} \\
& \quad+2 \nu_{y} e_{p}^{T} C_{c}^{T} K_{y}^{T} B_{c}^{T} P A_{r e f}^{-1} B_{c} \tilde{K}_{y} C_{c} e_{p}+2 e^{T} P B_{c} \tilde{K}_{y} \hat{x},
\end{aligned}
$$

which reduces to

$$
\begin{gathered}
\dot{V}\left(e, e_{p}, \tilde{K}_{x}, \tilde{K}_{y}\right)=-e^{T} Q e-e_{p}^{T} R e_{p}-\nu_{x} \hat{x}^{T} \tilde{K}_{x}^{T} B_{c}^{T} A_{r e f}^{-T} Q A_{r e f}^{-1} B_{c} \tilde{K}_{x} \hat{x}-\nu_{y} e_{p}^{T} C_{c}^{T} \tilde{K}_{y}^{T} B_{c}^{T} A_{r e f}^{-T} Q A_{r e f}^{-1} B_{c} \tilde{K}_{y} C_{c} e_{p} \\
+2 \nu_{x} \hat{x}^{T} K_{x}^{* T} B_{c}^{T} P A_{r e f}^{-1} B_{c} \tilde{K}_{x} \hat{x}+2 \nu_{y} e_{p}^{T} C_{c}^{T} K_{y}^{* T} B_{c}^{T} P A_{r e f}^{-1} B_{c} \tilde{K}_{y} C_{c} e_{p}+2 e_{p}^{T} W \underbrace{\left(\Delta A+\Delta B K_{y}^{*} C_{c}\right)}_{\Delta A_{a}} e_{p} \\
+2 e_{p}^{T} W \underbrace{\left(\Delta A+\Delta B K_{l q r}+\Delta B K_{x}^{*}\right)}_{\Delta A_{b}} \hat{x}+2 e_{p}^{T} W \Delta B \tilde{K}_{x} \hat{x}+2 e_{p}^{T} W \Delta B \tilde{K}_{y} C_{c} e_{p} .
\end{gathered}
$$

$\dot{V}\left(e, e_{p}, \tilde{K}_{x}, \tilde{K}_{y}\right)$ is bounded by

$$
\begin{aligned}
& \dot{V}\left(e, e_{p}, \tilde{K}_{x}, \tilde{K}_{y}\right) \leq-c_{1}\|e\|^{2}-\nu_{x} c_{2}\|\hat{x}\|^{2}\left\|\tilde{K}_{x}\right\|^{2}+2 \nu_{x} c_{3}\|\hat{x}\|^{2}\left\|\tilde{K}_{x}\right\|-\left(c_{4}-2 c_{7}\right)\left\|e_{p}\right\|^{2} \\
& \quad-\nu_{y} c_{5}\left\|e_{p}\right\|^{2}\left\|\tilde{K}_{y}\right\|^{2}+2 \nu_{y} c_{6}\left\|e_{p}\right\|^{2}\left\|\tilde{K}_{y}\right\|+2 c_{8}\|\hat{x}\|\left\|e_{p}\right\|+2 c_{9}\|\hat{x}\|\left\|e_{p} \mid\right\| \tilde{K}_{x}\left\|+2 c_{10}\right\| e_{p}\left\|^{2}\right\| \tilde{K}_{y} \|
\end{aligned}
$$

where $c_{1}=\lambda_{\min }(Q), c_{2}=\lambda_{\min }\left(B_{c}^{T} A_{r e f}^{-T} Q A_{\text {ref }}^{-1} B_{c}\right), c_{3}=\left\|B_{c}^{T} P A_{r e f}^{-1} B_{c}\right\|\left\|K_{x}^{*}\right\|, c_{4}=\lambda_{\min }(R), c_{5}=c_{2}\left\|C_{c}\right\|^{2}$, $c_{6}=\left\|B_{c}^{T} P A_{r e f}^{-1} B_{c}\right\|\left\|K_{y}^{*}\right\|\left\|C_{c}\right\|^{2}, c_{7}=\left\|W \Delta A_{a}\right\|, c_{8}=\left\|W \Delta A_{b}\right\|, c_{9}=\|W \Delta B\|$, and $c_{10}=c_{9}\left\|C_{c}\right\|$.

By using the inequality $2\|a\|\|b\| \leq\|a\|^{2}+\|b\|^{2}, \dot{V}$ can be bounded by

$$
\begin{array}{r}
\dot{V}\left(e, e_{p}, \tilde{K}_{x}, \tilde{K}_{y}\right) \leq-c_{1}\|e\|^{2}+c_{8}\|\hat{x}\|^{2}-\left(\nu_{x} c_{2}-c_{9}\right)\|\hat{x}\|^{2}\left\|\tilde{K}_{x}\right\|^{2}+2 \nu_{x} c_{3}\|\hat{x}\|^{2}\left\|\tilde{K}_{x}\right\| \\
-\left(c_{4}-2 c_{7}-c_{8}-c_{9}\right)\left\|e_{p}\right\|^{2}-\nu_{y} c_{5}\left\|e_{p}\right\|^{2}\left\|\tilde{K}_{y}\right\|^{2}+2\left(\nu_{y} c_{6}+c_{10}\right)\left\|e_{p}\right\|^{2}\left\|\tilde{K}_{y}\right\|,
\end{array}
$$


and after completing the square gives

$$
\begin{gathered}
\dot{V}\left(e, e_{p}, \tilde{K}_{x}, \tilde{K}_{y}\right) \leq-c_{1}\|e\|^{2}+\left(c_{8}+\frac{\nu_{x}^{2} c_{3}^{2}}{\nu_{x} c_{2}-c_{9}}\right)\|\hat{x}\|^{2}-\left(\nu_{x} c_{2}-c_{9}\right)\|\hat{x}\|^{2}\left(\left\|\tilde{K}_{x}\right\|-\frac{\nu_{x} c_{3}}{\nu_{x} c_{2}-c_{9}}\right)^{2} \\
-\left(c_{4}-2 c_{7}-c_{8}-c_{9}-\frac{\left(\nu_{y} c_{6}+c_{10}\right)^{2}}{\nu_{y} c_{5}}\right)\left\|e_{p}\right\|^{2}-\nu_{y} c_{5}\left\|e_{p}\right\|^{2}\left(\left\|\tilde{K}_{y}\right\|^{2}-\frac{\nu_{y} c_{6}+c_{10}}{\nu_{y} c_{5}}\right)^{2} .
\end{gathered}
$$

It can be shown that for $\dot{V} \leq 0$, the following inequalities must be satisfied:

$$
\begin{aligned}
c_{1} & >0 \\
c_{8}+\frac{\nu_{x}^{2} c_{3}^{2}}{\nu_{x} c_{2}-c_{9}} & <0 \\
\nu_{x} c_{2}-c_{9} & >0 \\
c_{4}-2 c_{7}-c_{8}-c_{9}-\frac{\left(\nu_{y} c_{6}+c_{10}\right)^{2}}{\nu_{y} c_{5}} & >0 .
\end{aligned}
$$

This analysis assumes that $C_{p}=C_{c}$, and $D_{p}=D_{c}$. Choosing $Q, R, \nu_{x}$, and $\nu_{y}$ to satisfy Eqs. (14a)-(14d) will produce a stable controller with adaptive augmentation.

Due to the conservative nature of the above Lyapunov analysis, it tends to produce small, and oftentimes non-existing stable boundaries for $\nu_{x}$ and $\nu_{y}$. Fortunately, the linear asymptotic property of OCM can be used to find feasible stable regions of $\nu_{x}$ and $\nu_{y}$.

\section{B. Asymptotic Stability Analysis}

The adaptive controller is most effective when adaption occurs quickly. This is achieved with the above controller by using large values for the adaptive learning rates $\Gamma_{x}$ and $\Gamma_{y}$. In practice, of course, this will lead to instability and/or large oscillations within the system, so it is not advisable. Thus, if the system is analyzed assuming an infinite learning rate, the results will be conservative since this corresponds to a zero time delay margin for MRAC. Assuming an infinite learning rate in Eqs. (6) and (7) produces

$$
\begin{aligned}
K_{x} \hat{x} & =-\frac{1}{\nu_{x}}\left(B_{c}^{T} A_{r e f}^{-T} P B_{c}\right)^{-1} B_{c}^{T} P e \\
K_{y}(y-\hat{y}) & =-\frac{1}{\nu_{x}}\left(B_{c}^{T} A_{r e f}^{-T} P B_{c}\right)^{-1} B_{c}^{T} P e
\end{aligned}
$$

and the augmented control law from (5) becomes

$$
u^{\infty}=K_{l q r} \hat{x}+\underbrace{\left(-\left(B_{c}^{T} A_{r e f}^{-T} P B_{c}\right)^{-1} B_{c}^{T} P\left(\frac{1}{\nu_{x}}+\frac{1}{\nu_{y}}\right)\right)}_{K_{a d}^{\infty}} e .
$$

Combining the true plant model of Eq. (1), the observer model of Eq. (2), the reference model of Eq. (4), and the adaptive augmented control law with asymptotic adaptive learning rate gain of Eq. (17) produces the closed loop system

$$
\frac{d}{d t}\left[\begin{array}{c}
x \\
\hat{x} \\
x_{r e f}
\end{array}\right]=\underbrace{\left[\begin{array}{ccc}
A_{p} & B_{p}\left[K_{l q r}-K_{a d}^{\infty}\right] & B_{p} K_{a d}^{\infty} \\
L C_{p} & A_{c}-L C_{c}+\left[B_{c}+L \Delta D\right]\left[K_{l q r}-K_{a d}^{\infty}\right] & {\left[B_{c}+L \Delta D\right] K_{a d}^{\infty}} \\
0 & 0 & A_{r e f}
\end{array}\right]}_{A_{c l}^{\infty}}\left[\begin{array}{c}
x \\
\hat{x} \\
x_{r e f}
\end{array}\right] .
$$

Thus, the stability of the system with adaptive augmentation can be estimated by the above asymptotic (with respect to $\Gamma_{x}$ and $\Gamma_{y}$ ) linear system. This analysis is less conservative than the earlier Lyapunov analysis, but it still represents the worst case scenario for margins at a theoretical infinite adaptive gain. For finite values of $\Gamma_{x}$ and $\Gamma_{y}$, the actual stable regions will be larger. Thus, the actual regions for stable $\nu_{x}$ and $\nu_{y}$ will be greater than what Eq. (18) predicts. Note that the above matrix includes the potential for $C_{p} \neq C_{c}$, and $D_{p} \neq D_{c} \neq 0$.

In the following section, this result will be used on some notional toy problems to demonstrate some of analytical features of the OCM and its asymptotic property. 


\section{Toy Problems}

In this section two numerical examples are used to provide some insight into the analytical and performance properties of OCM relative to signal delay, plant perturbation, and nominal controller attributes.

\section{A. Scalar System}

The following scalar system is used for feedback and estimation LQG control:

$$
\begin{aligned}
& \dot{x}=x-3 u \\
& y=x
\end{aligned}
$$

Assume no plant perturbation, and with $q_{l q r}=r_{l q r}=1, q_{e s t}=r_{e s t}=1$, which results in LQR feedback gain of $k_{l q r}=1.3874$, and an estimator gain of $l=2.4142$. The controller is a simple regulator, and in the adaptive case, the reference states are zero. Thus, $e=-\hat{x}$, and the asymptotic OCM control law is $u^{\infty}=k^{*} \hat{x}$ where $k^{*}=k_{l q r}+\left(\frac{1}{\nu_{x}}+\frac{1}{\nu_{y}}\right) \frac{a_{r e f}}{b} . A_{c l}^{\infty}$ for this simple scalar problem reduces to

$$
A_{c l}^{\infty}=\left[\begin{array}{cc}
a & b k^{*} \\
l c & a-l c+b k^{*}
\end{array}\right]
$$

It is clear that for this system large $\nu$ values result in $k^{*} \approx k_{l q r}$, and as either $\nu_{x}$ and/or $\nu_{y} \rightarrow 0, k^{*} \rightarrow \infty$. For this system, the asymptotic property of the OCM adaptive controller predicts stable control for all values of $\nu_{x}$ and $\nu_{y}$, however, further insight can be gained into the relationship between $\nu$ and time delay margin robustness.

\section{Time Delay Margin Analysis}

We now consider the case where the control signal $u(t)$ applied to the plant and the observer/controller is delayed by $\tau$. We define the time delay margin $t_{d}$ as the maximum permissible value of $\tau$ before closed loop system stability is lost. In this situation, the above closed loop system matrix becomes

$$
A_{c l}^{\infty}(\omega \tau)=\left[\begin{array}{cc}
a & b k^{*} e^{-j \omega \tau} \\
l c & a-l c+b k^{*} e^{-j \omega \tau}
\end{array}\right]
$$

Here we have used the fact that $u(t-\tau)=k^{*} \hat{x}(t-\tau)$. Both the gain crossover frequency $\omega_{g c}$ and the time delay margin $t_{d}$ can be found by solving for the roots of the characteristic equation as follows:

$$
\operatorname{det}\left(A_{c l}^{\infty}\left(\omega_{g c} t_{d}\right)\right)=\left(j \omega_{g c}-a\right)\left(j \omega_{g c}-a-b k^{*} e^{-j \omega_{g c} t_{d}}+a_{0}\right)-l c b k^{*} e^{-j \omega_{g c} t_{d}}=0
$$

where $a_{0}=l c-a$. This yields the following system of equations:

$$
\begin{aligned}
\omega_{g c}^{2}+a_{0} a & =-b k^{*}\left(\omega_{g c} \sin \left(\omega_{g c} t_{d}\right)+a_{0} \cos \left(\omega_{g c} t_{d}\right)\right) \\
\omega_{g c}\left(a+a_{0}\right) & =-b k^{*}\left(\omega_{g c} \cos \left(\omega_{g c} t_{d}\right)-a_{0} \sin \left(\omega_{g c} t_{d}\right)\right) .
\end{aligned}
$$

The above is combined as

$$
\omega_{g c}^{4}+\left(a^{2}+a_{0}^{2}-\left(b k^{*}\right)^{2}\right) \omega_{g c}^{2}+\left(a_{0} a\right)^{2}-\left(a_{0} b k^{*}\right)^{2}=0
$$

which can be solved for $\omega_{g c}$. Multiplying (24) and (25) by $a_{0}$ and $\omega_{g c}$ respectively gives the time delay margin as

$$
t_{d}=\frac{1}{\omega_{g c}} \cos ^{-1}\left(\frac{a}{b k^{*}}\right) .
$$

For the case where either $\nu_{x}$ or $\nu_{y} \rightarrow 0, k^{*} \rightarrow \infty$. Equation (26) can be solved with large $k^{*}$ approximately as

$$
\omega_{g c}^{2} \approx \frac{\left(b k^{*}\right)^{2}+b k \sqrt{\left(b k^{*}\right)^{2}+4 a_{0}^{2}}}{2},
$$


which shows that $\omega_{g c} \rightarrow \infty$, and from equation (27), $t_{d} \rightarrow 0$. Thus, as either $\nu_{x}$ or $\nu_{y} \rightarrow 0$, the time delay margin for delay on both sides of the plant goes to zero. In other words, the asymptotic stability analysis shows that as OCM control approaches simple MRAC control, robustness is lost.

A plot of the relation between $\nu_{x}=\nu_{y}$ and $t_{d}$ is shown in Fig. 1 for this system based on the exact solution of equations (26) and (27). This will now be used to accurately predict loss of stability due to time delay.

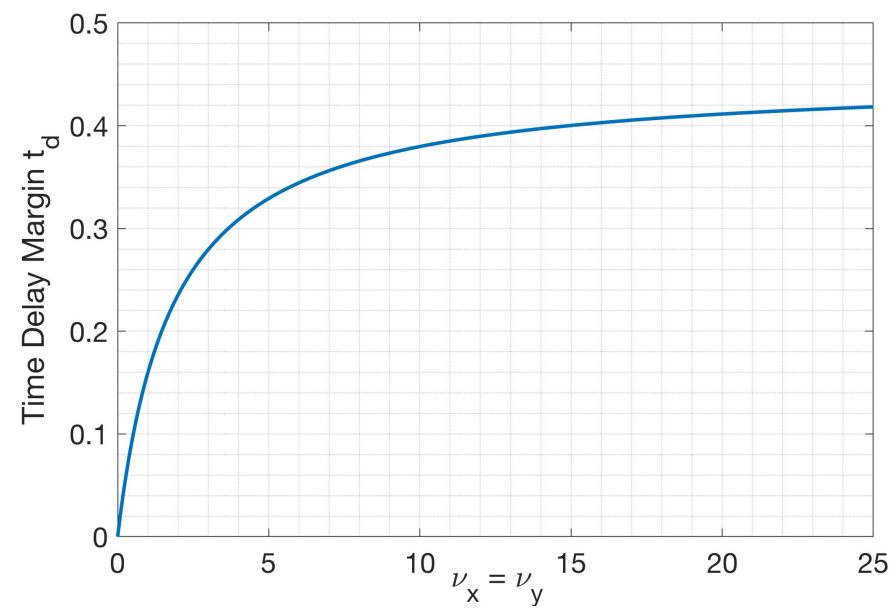

Figure 1. Time delay margin vs. $\nu_{x}=\nu_{y}$.

\section{Time Delay Simulation}

Setting $\Gamma_{x}=\Gamma_{y}=1 \times 10^{6}$, the scalar system is simulated under a variety of circumstances. In Fig. 2, using basic MRAC control and no time delay, the system is shown to be regulated, but exhibits transient high frequency oscillations. Next, a small time delay of $\tau=0.001 \mathrm{~s}$ is added to the plant and controller inputs as represented in equation 22. As expected, the MRAC controller goes unstable. Using OCM control and setting $\nu_{x}=\nu_{y}=1$, we see that the delay is easily handled. In fact, using equation (23) without any approximation (since for $\nu_{x}=\nu_{y}=1, b k \ngtr a$ ), the asymptotic OCM analysis predicts a delay margin of $t_{d}=0.160$ s. (See Fig. 1.) This onset of instability is shown in purple in Fig. 2.

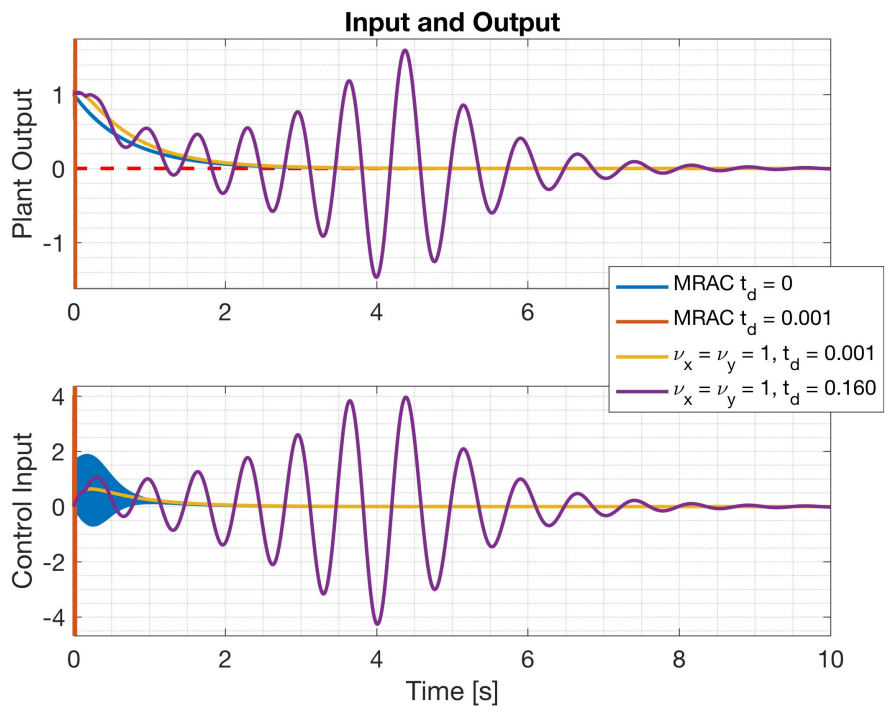

Figure 2. Simulation results for basic MRAC versus OCM with delay at both the plant and controller inputs. 


\section{B. 2-state System}

The above analysis on a simple unperturbed system demonstrates the improvement that OCM provides over standard MRAC in terms of time delay robustness. As system complexity increases, and the potential of plant perturbation exists, there is a limit to the benefit that OCM can provide. The asymptotic property of OCM can be used to find those limits. In this section we observe how the stable regions (relative to $\nu$ ) are affected by plant perturbations and nominal controller design.

Consider the model of the following unstable 2-state system:

$$
A_{c}=\left[\begin{array}{cc}
1 & 2 \\
1 & 0.5
\end{array}\right], B_{c}=\left[\begin{array}{l}
1 \\
0
\end{array}\right], C_{c}=\left[\begin{array}{ll}
0 & 1
\end{array}\right], D_{c}=0 .
$$

The actual plant is perturbed according to $A_{p}=\left(1+\delta_{A}\right) A_{c}, B_{p}=\left(1+\delta_{B}\right) B_{c}$, and $C_{p}=\left(1+\delta_{C}\right) C_{c}$. Before analyzing stable regions for tuning the adaptive OCM control, it is worth exploring the performance it offers in simulation.

\section{Perturbed Plant Simulation}

First, a baseline LQG regulator control law of $u=K_{l q r} \hat{x}$ is calculated based on the controller model of (29). Figure 3 shows the simulation results of both the unperturbed as well as the perturbed system with $\delta_{A}=0.05, \delta_{B}=-0.05$, and $\delta_{C}=-0.05$. The initial condition of the plant is set to $x(0)=[10-1]^{T}$. As expected, the unperturbed plant output is stabilized and regulated to zero, but the perturbation of the off-nominal plant is sufficient to result in instability.

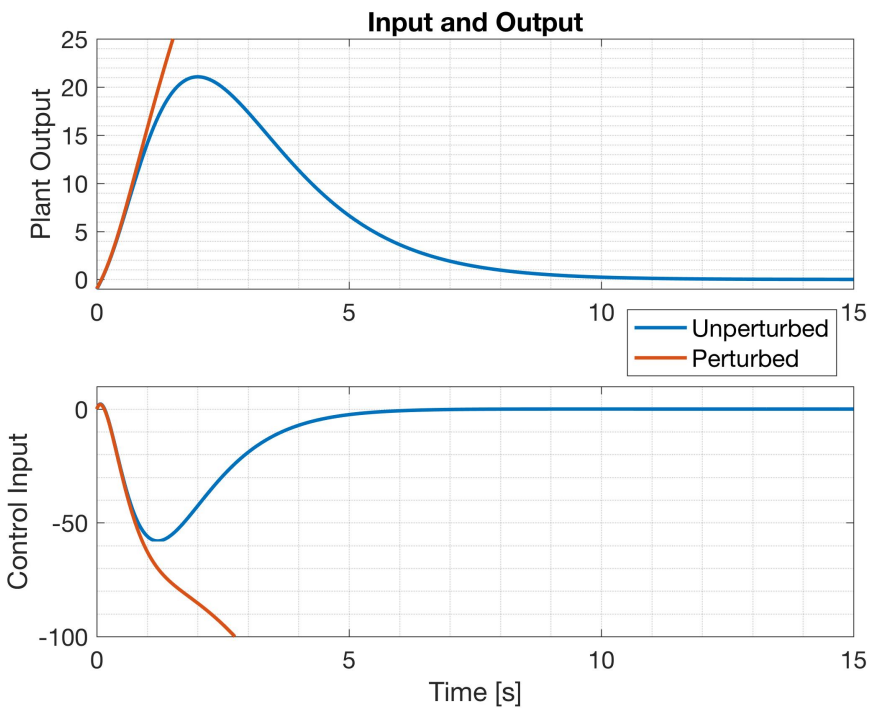

Figure 3. Baseline control of the unperturbed and perturbed system with $\delta_{A}=0.05, \delta_{B}=-0.05, \delta_{C}=-0.05$. 


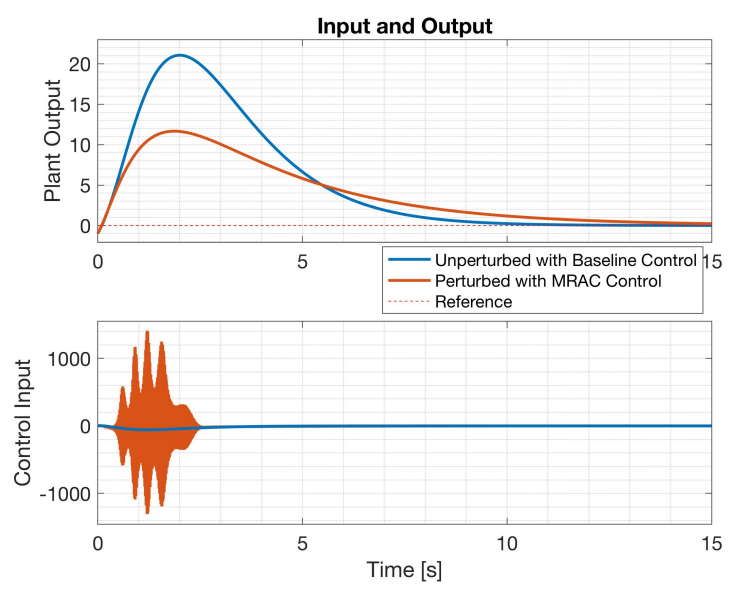

(a) Input and Output
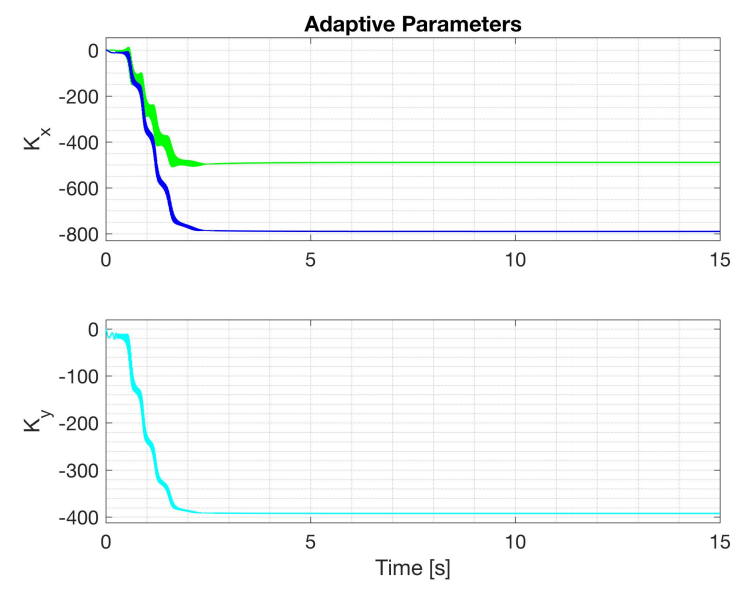

(b) Adaptive parameters of the MRAC controller

Figure 4. Baseline LQG control of the unperturbed system compared to the perturbed system with adaptive MRAC augmented control.

The hope is that with the use of adaptive control, this perturbed plant can be stabilized. Thus, adaptive control is added to the nominal controller according to Eq. (5). First, $K_{x}(t)$ and $K_{y}(t)$ adapt with the standard MRAC adaptive laws, which are achieved by setting $\nu_{x}=\nu_{y}=0$ in Eqs. (6) and (7). Again, since this is a regulator system, $e=-\hat{x}$. For fast adaptation, $\Gamma_{x}=1 \times 10^{4} I_{2}$, and $\Gamma_{y}=1 \times 10^{4}$. It is seen in Fig. 4 that the MRAC adaptive augmentation stabilizes the perturbed plant. However, the high frequency oscillations shown in the control input signal and in the adaptive gains reveal the system is not robust during the transient phase of adaption, and certainly would not be tenable for any real system. Thus, $\nu_{x}$ and $\nu_{y}$ are increased to improve performance.

Figure 5(a) on the following page compares the performance of the system augmented with basic MRAC control to four others with increasing values of $\nu_{x}$ and $\nu_{y}$. All results use the same perturbed system with identical initial conditions and adaptive learning rate values of $\Gamma_{x}$ and $\Gamma_{y}$ from above. Setting $\nu_{x}=\nu_{y}=0.01$ produces quick adaptation nearly identical to the MRAC results but without the harmful oscillations. The adaptive gains for this scenario are plotted in Fig. 5(b) on the next page revealing that the controller adapts quickly, and the gains converge to much smaller magnitudes than in the MRAC scenario depicted in Fig. 4(b). As values of $\nu$ are increased, note that the tracking performance suffers. For $\nu_{x}=\nu_{y}=1.175$, the system is neutrally stable, and at $\nu_{x}=\nu_{y}=1.5$ the system is unstable. In the next section, it is shown that this loss of steady state stability due to $\nu$ can be accurately predicted using the asymptotic properties of OCM discussed earlier. It should be noted that it is not always the case that increasing $\nu$ leads to instability. The GTM model discussed in Section V exhibits the opposite trend in terms of $\nu$, where the unstable region is a lower bound on $\nu$. 


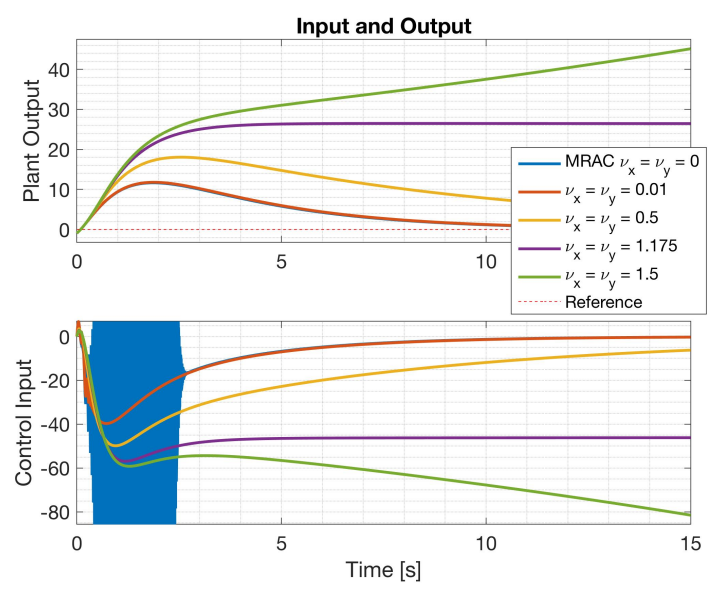

(a) MRAC and OCM control of the perturbed system with increasing $\nu_{x}, \nu_{y}$
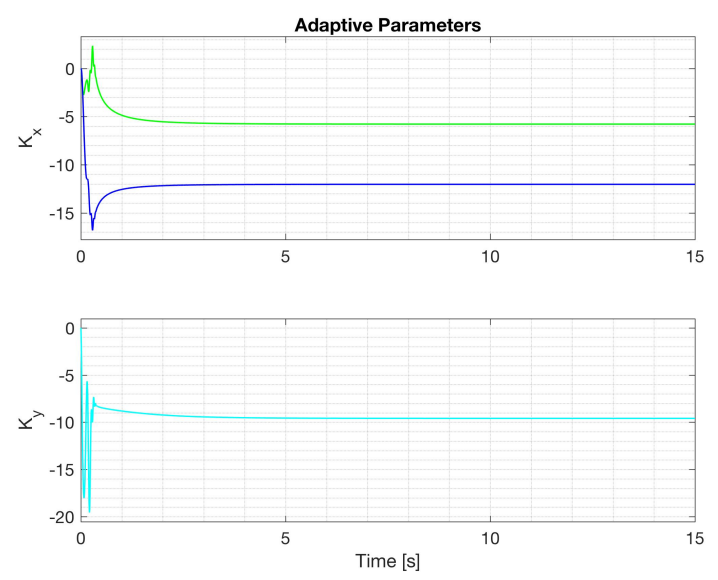

(b) Adaptive parameters of the OCM controller for $\nu_{x}=\nu_{y}=$ 0.01

Figure 5. MRAC versus OCM control simulated results.

\section{2. $\quad \nu$ Versus Perturbation}

Earlier, the asymptotic closed loop system matrix was derived for a system using LQG control with OCM augmentation. This analysis is performed assuming the actual true plant is known along with the controller's notion of the plant. In practice this would not be the case, however if bounds or estimates for $\Delta A, \Delta B, \Delta C$, $\Delta D$ are known, this analytical method can be quite useful for identifying bounds for $\nu$ that produce stable controllers. $\Gamma$ can then be tuned to provide the desired adaptation rate for practical implementation.

Again, using the toy problem of (29), and the same nominal controller with OCM augmentation, $\delta_{A}, \delta_{B}$, and $\delta_{C}$ are altered and the asymptotic closed loop system matrix of Eq. (18) can be evaluated through a range of $\nu_{x}$ and $\nu_{y}$. Figure 6 shows the regions where $A_{c l}^{\infty}$ is stable (shown in blue) for various values of $\delta_{A}$, $\delta_{B}$, and $\delta_{C}$ perturbation. Note that Fig. $6(\mathrm{~b})$ depicts the perturbation case used above in simulation, and that these regions agree with the simulation results. Specifically, neutral stability is predicted to occur at $\nu_{x}=\nu_{y}=1.175$ (identified in the figure), which lies on the boundary of the stable/unstable regions.

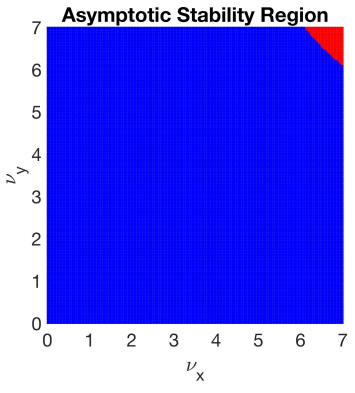

(a) $\delta_{A}=0.035, \delta_{B}=-0.035, \delta_{C}=$ $-0.035$

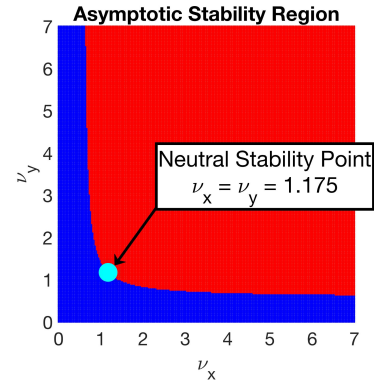

(b) $\delta_{A}=0.050, \delta_{B}=-0.050, \delta_{C}=$ $-0.050$

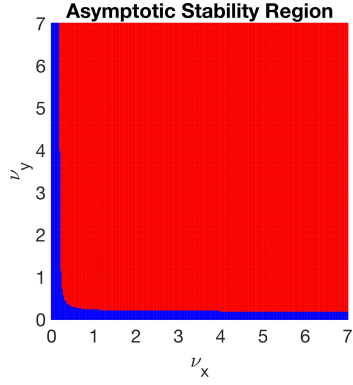

(c) $\delta_{A}=0.065, \delta_{B}=-0.065, \delta_{C}=$ $-0.065$

Figure 6. Stable boundaries for $\nu_{x}$ and $\nu_{y}$ (shown in blue) for various $\delta_{A}, \delta_{B}, \delta_{C}$ values.

The difference between the controller's notion of the plant and the true plant can be quantified using the $\mathcal{H}_{2}$ norm of the difference between the transfer functions of both models. Since the models may be unstable, the $\mathcal{H}_{\sigma-2}$ norm is defined as

$$
\|G(s)\|_{\sigma-2}=\|G(s+\sigma)\|_{2}=\left\|C[(s+\sigma) I-A]^{-1} B+D\right\|_{2}
$$

where $\sigma>0$ is chosen so that it is greater than the largest positive real part of all eigenvalues of $A$. Another example of how this technique is used for quantifying the difference between two LTI models can be found in Ref. 12. 
The relation between the size of the plant perturbation and the size of the stable region for $\nu$ can now be observed by plotting the max stable value of $\nu_{x}=\nu_{y}$ against the norm of the difference between the two models $\left\|G_{p}(s)-G_{c}(s)\right\|_{\sigma-2}$. It is found that for system (29), $\delta_{B}$ has the greatest effect on this difference. Therefore, in Fig. 7 keeping $\delta_{A}=\delta_{C}=\delta_{D}=0, \delta_{B}$ is decreased from 0 and the max stabilizing values of $\nu_{x}=\nu_{y}$ are found. It is not surprising to see that there is a limit to what stability may be recovered by the adaptive OCM controller given the size of the plant perturbation.

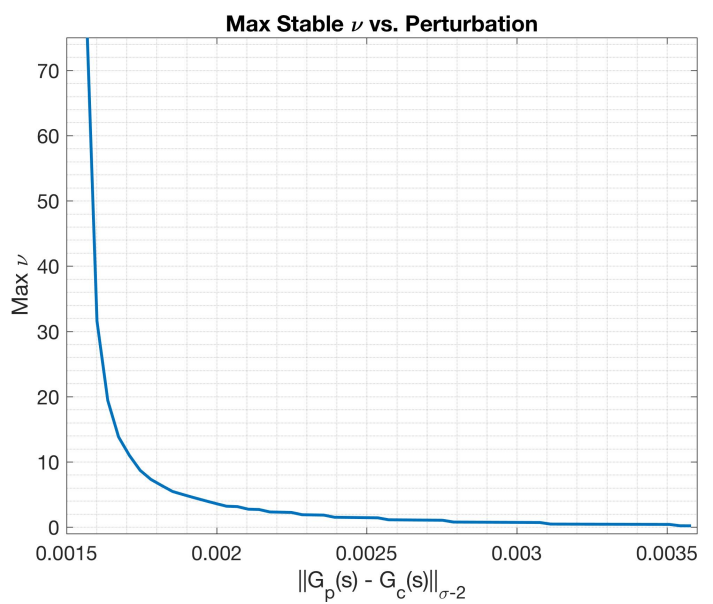

Figure 7. Maximum value of $\nu_{x}=\nu_{y}$ vs. $\left\|G_{p}(s)-G_{c}(s)\right\|_{\sigma-2}$ found by decreasing $\delta_{B}$ from $0 \rightarrow-0.2$ of system (29).

\section{3. $\quad$ V Versus Baseline LTR Control}

It is well known that while LQR control has guaranteed phase and gain margin properties, LQG control, in general does not. ${ }^{13}$ This can be partially remedied by the use of the loop transfer recovery (LTR) design method. ${ }^{14}$ Simply stated, the state noise covariance $Q_{e s t}$ is altered via a tunable parameter $\rho$ according to

$$
Q_{e s t}=Q_{0}+\frac{1}{\rho} B_{c} B_{c}^{T}
$$

so that the resulting system approaches an "LQR-like" system as $\rho \rightarrow 0$. Realistically, this procedure is limited by the potential of amplifying system output noise because as $\rho \rightarrow 0,\|L\| \rightarrow \infty$. The LTR technique is a valuable tool for improving the robustness of the baseline controller when applied with care to not significantly amplify output/sensor noise. Similar techniques of improving baseline controller robustness have recently been developed. See, for example, Ref. 15.

Using the toy problem of (29), and the plant perturbation of $\delta_{A}=0.05, \delta_{B}=-0.05, \delta_{C}=-0.05$, Fig. 8 on the following page shows that the baseline controller (without the adaptive portion) tuned via LTR with $\rho=1 \times 10^{-4}$ can stabilize the plant. However, the resulting observer gains are $L=\left[\begin{array}{lll}117 & 16\end{array}\right]^{T}$, substantially increasing the risk of amplifying output noise. The non LTR controller with augmented OCM, on the other hand, results in a stable system that uses observer gains of $L=[8.34 .7]^{T}$, with adaptive gains staying well bounded and relatively small as seen in Fig. $5(\mathrm{~b})$ on the previous page.

It is interesting to observe how the stable region of $\nu_{x}$ and $\nu_{y}$ is altered by the LTR tuning parameter $\rho$. Figure 9 on the following page shows how increasing the robustness of the baseline LQG controller with LTR also scales the size of the stable region for $\nu_{x}$ and $\nu_{y}$. The 2-norm of the observer gain is also shown, demonstrating that the use of LTR on the baseline controller can potentially ease the tuning and implementation of a stable adaptive controller, but at the expense of higher output noise amplification.

In the following section, these above analytical results are used to implement an OCM controller an adaptive flutter suppression controller. 

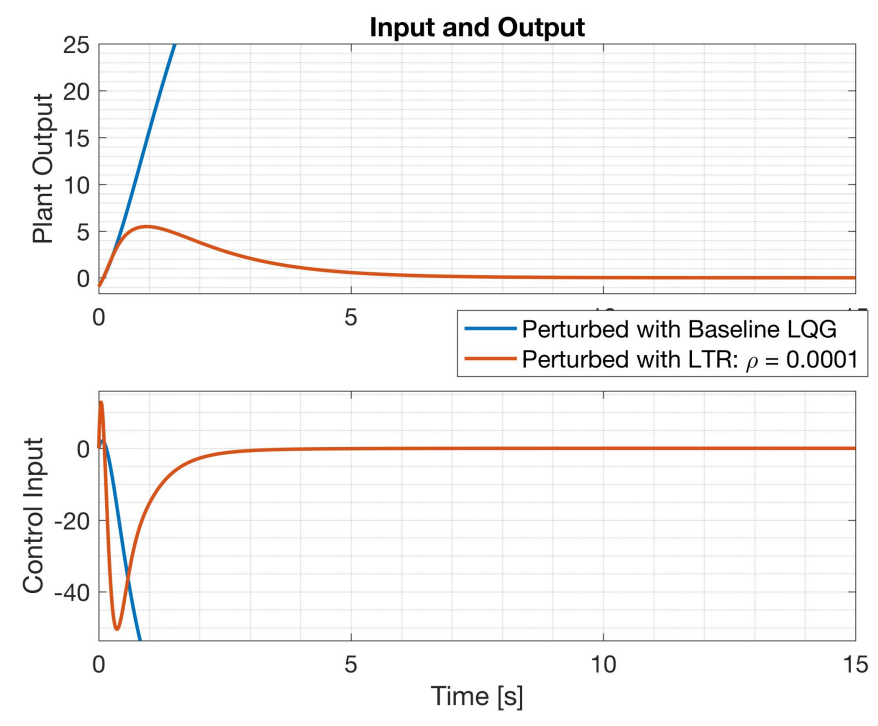

Figure 8. Non adaptive control of the perturbed system with and without LTR.

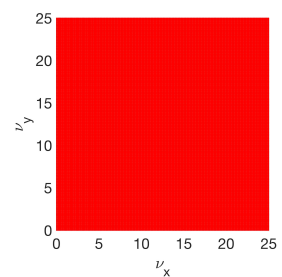

(a) No LTR, $\|L\|=9.53$

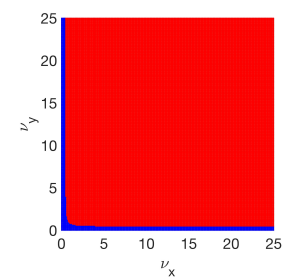

(b) $\rho=0.05,\|L\|=13.1$

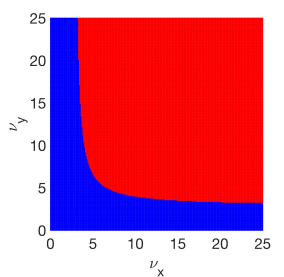

(c) $\rho=0.01,\|L\|=19.5$

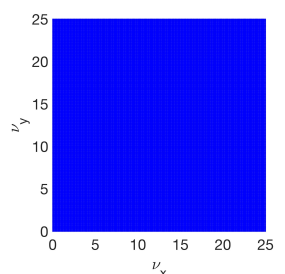

(d) $\rho=0.001,\|L\|=44.1$

Figure 9. Stable regions for $\nu_{x}$ and $\nu_{y}$ (shown in blue) with plant perturbation of $\delta_{A}=-0.15, \delta_{B}=0.15$ for various LTR values of $\rho$.

\section{GTM Flutter Suppression}

As aircraft manufacturers seek ways to reduce weight and increase efficiency, the resulting loss of structural rigidness may decrease the aerodynamic performance, leading to increased drag and reduced flutter margins. In recent years, research has been investigating Performance Adaptive Aeroelastic Wing (PAAW) technology, which incorporates novel control surface designs and control algorithms to actively shape the wing and mitigate these issues. One proposed technology is the Variable Camber Continuous Trailing Edge Flap (VCCTEF) actuator. ${ }^{16}$ This is a concept developed under NASA's Advanced Air Transport Technology (AATT) project. For the Generic Transport Model (GTM) considered here, which is similar to a Boeing 757 airframe, the trailing edge is modeled to consist of 16 individually controlled 3 -segment flaps. Each flap is connected to its neighbor using a flexible material to avoid drag producing gaps. (See Fig. 10 on the next page). This provides the ability to change the wing shape in flight in order to achieve a variety of control objectives. Ref. 17 presents an aeroservoelastic (ASE) model of the GTM with VCCTEF, along with simulation results of a multi-objective optimal controller that provides drag reduction, maneuver load reduction, and flutter suppression. In this paper, the longitudinal version of the GTM ASE model is used to design a flight path angle tracking controller with adaptive flutter suppression. The bending and torsional stiffness parameters of the model have been reduced by $50 \%$ in order to simulate a softer wing with a lower flutter boundary. Similar work can be found in Refs. 11 and 18. 


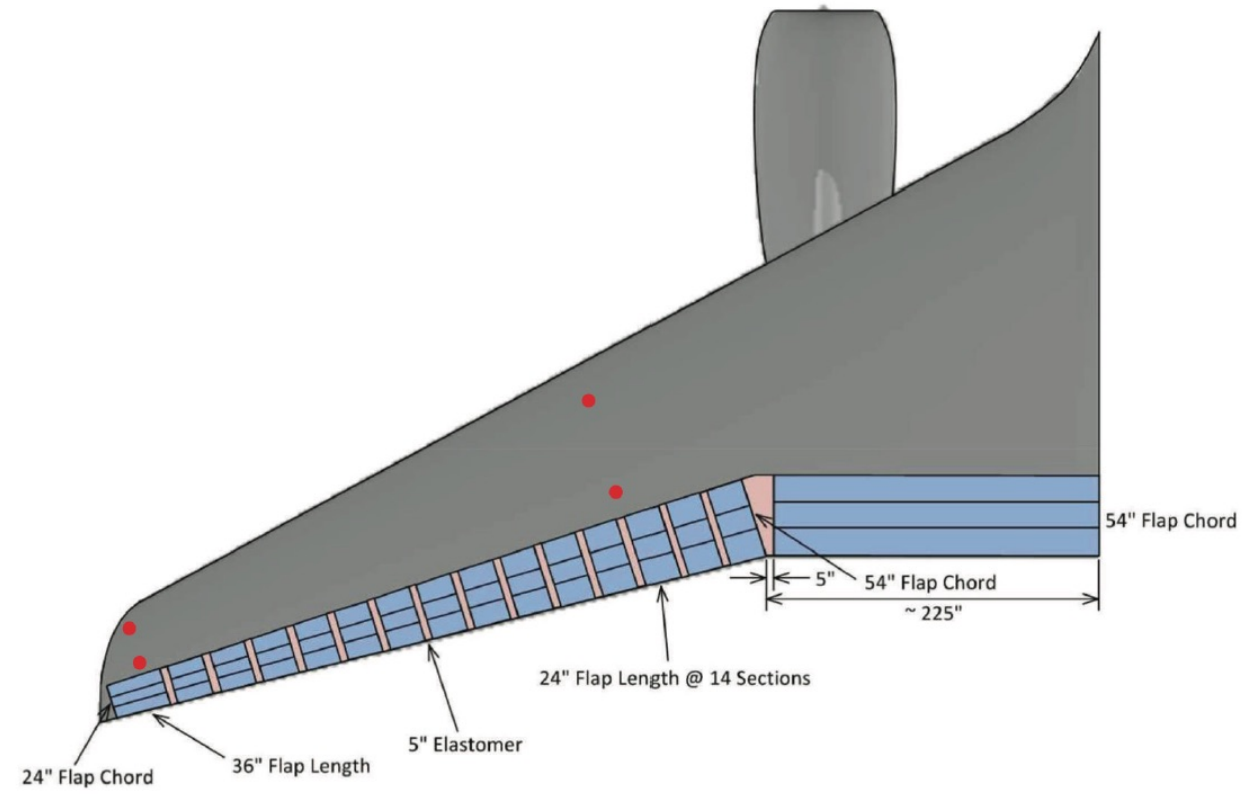

Figure 10. The VCCTEF wing, showing 1 inboard and 15 outboard 3-segment flaps.

\section{A. Model Specifics}

The full longitudinal GTM model is generated for a trim condition based on altitude, Mach, and fuel load. Since the full model consists of over 1300 states, it is beneficial for control and simulation purposes to reduce the model using significantly fewer structural modes while maintaining a reasonable level of fidelity. Figure 11 on the following page shows the flutter boundary between the stable and unstable regions for the softened model at $80 \%$ fuel load. The plot indicates that a model containing only the first 10 modes has a very similar flutter boundary to the full model. Thus, this model at $80 \%$ fuel load will be used for simulation.

As above, we distinguish the model used for control and observer design from the one used as the true plant on which the simulation is conducted using the subscripts $c$ and $p$ respectively. For both models, aeroelastic lag states are included for the first 10 modes using the R.T. Jones method resulting in 6 states per mode. Also, no control surface actuator dynamics or sensor dynamics are included. Three rigid body states $\alpha, q$, and $\theta$ are assumed to be perfectly known. For this study both the controller/observer model and the plant model contain 63 states $\left(n_{c}=n_{p}=63\right)$. However, the two models may be separated by trim condition, as well as other linear perturbations prescribed as they are to the toy problem above. Two vertical accelerometers placed $5 \mathrm{ft}$ inboard from the tip of each wing $2 \mathrm{ft}$ forward and aft of the elastic axis are used to augment the observer's estimation of the elastic states used for the flutter controller.

In this analysis, the tracking control is allocated to the elevator only, and the VCCTEF flaps are reserved for flutter suppression using an LQG controller augmented with adaptive MRAC OCM. With this control allocation, the plant model can be prescribed as

$$
\begin{aligned}
& \dot{x}=A_{p} x+\left[\begin{array}{ll}
B_{p}^{r b} & B_{p}^{f}
\end{array}\right]\left[\begin{array}{c}
u^{r b} \\
u^{f}
\end{array}\right] \\
& y=\left[\begin{array}{c}
C_{p}^{t} \\
C_{p}^{a c c e l}
\end{array}\right] x+\left[\begin{array}{cc}
0 & 0 \\
D_{p}^{a c c e l} l_{r b} & D_{p}^{a c c e l} l_{f}
\end{array}\right]\left[\begin{array}{c}
u^{r b} \\
u^{f}
\end{array}\right]
\end{aligned}
$$

Here, $u^{r b}$ refers to the tracking control input to the elevator, and $u^{f}$ refers to the flutter control input to the VCCTEF flaps. The VCCTEF flap design envisions each flap being connected to its neighboring flap with an elastomeric material. Thus, a $\pm 2^{\circ}$ limit between a flap and its neighbor should be enforced. Here, we have simplified the control surfaces of the original model by using single-segment flaps. Also, since the 


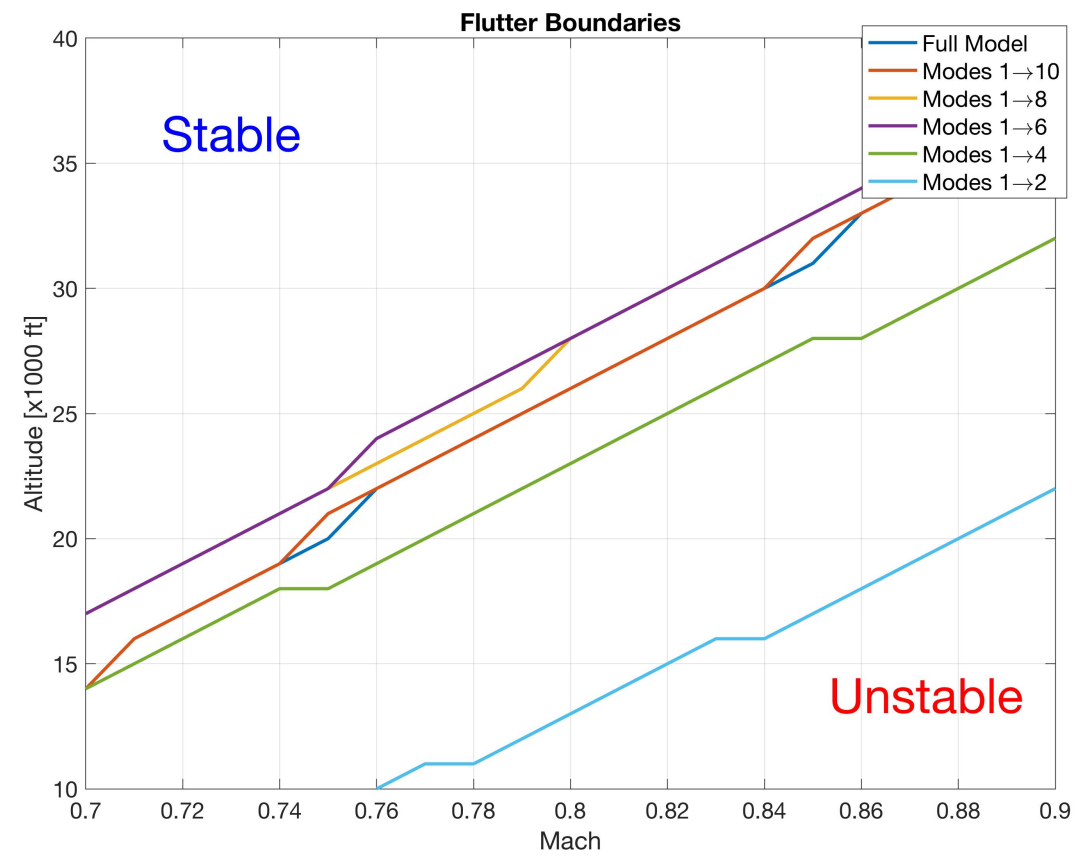

Figure 11. Stability flutter boundary regions of the longitudinal model with $80 \%$ fuel load.

flaps nearest the tips have a greater affect on flutter, this study only utilizes the last four flaps of the model referred to hereafter as flaps 1 through 4 . In order to conveniently enforce limits on the input applied to the plant model, the flutter control consists of 4 channels denoted as

$$
u^{f}=\left[u_{1}^{f}, \delta_{2}^{f}, \delta_{3}^{f}, \delta_{4}^{f}\right]
$$

$u_{1}^{f}$ commands the absolute deflection of the first flap, limited to $\pm 35^{\circ} . \quad \delta_{2}^{f}$ commands the second flap in terms of the difference from the first flap limited to $\pm 2^{\circ}$, and so on. Note that these limits are not taken into consideration by the controller. Instead, if the control requests input that exceeds them, they are capped at the limits prior to being applied to the true plant model.

The plant output described in equation of Eq. 32 consists of the tracked flight path angle $\gamma$, whose matrices are indicated by the $t$ superscript, and the two accelerometer outputs indicated by the accel superscript.

\section{B. Tracking Control}

The servomechanism LQG tracking controller is derived from the model by adding a state that equates to the integral of the tracking error defined as

$$
x_{i n t}=\int_{0}^{t}\left(\gamma(\tau)-\gamma_{c}(\tau)\right) d \tau
$$

where

$$
\gamma=C_{c}^{t} x
$$

The $c$ subscript, consistent with the above notation, denotes that this is the controller's notion of the plant on which the control gains will be computed. The extended servomechanism system is

$$
\underbrace{\left[\begin{array}{c}
\dot{x}_{\text {int }} \\
\dot{x}
\end{array}\right]}_{\dot{x}_{w}}=\underbrace{\left[\begin{array}{cc}
0 & C_{c}^{t} \\
0 & A_{c}
\end{array}\right]}_{A_{w}^{r b}} \underbrace{\left[\begin{array}{c}
x_{i n t} \\
x
\end{array}\right]}_{x_{w}}+\underbrace{\left[\begin{array}{c}
0 \\
B_{c}^{r b}
\end{array}\right]}_{B_{w}^{r b}} u^{r b}+\underbrace{\left[\begin{array}{c}
-1 \\
0
\end{array}\right]}_{B_{r}} \gamma_{c}
$$


where $x$ includes rigid body states, aeroelastic states, and any other states included in the controller's model. $u^{r b}$ is the input to the elevator, with $B_{c}^{r b}$ the portion of the $B_{c}$ matrix corresponding to that input. The $\mathrm{LQR}$ state feedback tracking control gains are computed by defining a cost function according to

$$
\begin{aligned}
J & =\lim _{t_{f} \rightarrow \infty} \frac{1}{2} \int_{0}^{t_{f}}\left\{q_{p}\left(\gamma-\gamma_{c}\right)^{2}+q_{i n t} x_{i n t}^{2}+u^{r b^{T}} R u^{r b}\right\} d t \\
& =\lim _{t_{f} \rightarrow \infty} \frac{1}{2} \int_{0}^{t_{f}}\left\{\left(C_{c}^{t} x-\gamma_{c}\right)^{T} q_{p}\left(C_{c}^{t} x-\gamma_{c}\right)+q_{i n t} x_{i n t}^{2}+u^{r b^{T}} R u^{r b}\right\} d t \\
& =\lim _{t_{f} \rightarrow \infty} \frac{1}{2} \int_{0}^{t_{f}}\left\{x_{w}^{T} \bar{Q} x_{w}-2 x_{w}^{T} T r+q_{p} r^{2}+u^{r b} R u^{r b}\right\} d t
\end{aligned}
$$

where $\bar{Q}=\left[\begin{array}{ll}0 & C_{c}^{t}\end{array}\right]^{T} q_{p}\left[\begin{array}{ll}0 & C_{c}^{t}\end{array}\right]+Q_{i n t}, Q_{i n t}=\left[\begin{array}{cc}q_{\text {int }} & 0 \\ 0 & 0\end{array}\right], T=\left[\begin{array}{ll}0 & C_{c}^{t}\end{array}\right]^{T} q_{p}$, and $q_{p}$ is the proportional tracking error weight, and $q_{\text {int }}$ is the integral tracking error weight.

The LQR control law for rigid body tracking is defined as

$$
u_{l q r}^{r b}=\underbrace{-R^{-1} B_{w}^{r b^{T}} P^{r b}}_{K_{x}^{r b}} x_{w}+\underbrace{-R^{-1} B_{w}^{r b} V}_{K_{r}^{r b}} \gamma_{c}
$$

where $P^{r b}$ is the solution to the standard Riccati equation

$$
A_{w}^{r b^{T}} P^{r b}+P^{r b} A_{w}^{r b}-P^{r b} B_{w}^{r b} R^{-1} B_{w}^{r b} P^{r b}+\bar{Q}=0
$$

and $V$ is found according to

$$
V=\left(A_{w}^{r b^{T}}-P^{r b} B_{w}^{r b} R^{-1} B_{w}^{r b}\right)^{-1}\left(T-P^{r b} B_{r}\right) .
$$

\section{Observer Design}

For this instantiation, we assume that the rigid body states are known, and all other states must be observed. This results in the LQG control law for tracking control

$$
u_{l q g}^{r b}=K_{x}^{r b}\left[\begin{array}{c}
x_{i n t} \\
x_{r b} \\
\hat{x}_{e}
\end{array}\right]+K_{r}^{r b} \gamma_{c}
$$

where $\hat{x}_{e}$ represents the estimated states. A Kalman estimator is used that estimates all states according to the controller's plant model, but the rigid body states are currently unused. The estimated state dynamics are defined as

$$
\dot{\hat{x}}=A_{c} \hat{x}+\underbrace{\left[\begin{array}{ll}
B_{c}^{r b} & B_{c}^{f}
\end{array}\right]}_{B_{c}}\left[\begin{array}{c}
u^{r b} \\
u^{f}
\end{array}\right]+L(y-\hat{y})
$$

where $B_{c}^{f}$ is the portion of the $B_{c}$ matrix corresponding to the inputs used for flutter control $u^{f}$. The observer output consists of both the tracking output $\gamma$ and accelerometer outputs. The estimated output can be decomposed according to+

$$
\hat{y}=\underbrace{\left[\begin{array}{c}
C_{c}^{t} \\
C_{c}^{a c c e l}
\end{array}\right]}_{C_{c}} \hat{x}+\underbrace{\left[\begin{array}{cc}
0 & 0 \\
D_{c}^{a c c e l} l_{r b} & D_{c}^{a c c e l} l_{f}
\end{array}\right]}_{D_{c}}\left[\begin{array}{c}
u^{r b} \\
u^{f}
\end{array}\right]
$$

and $L$ is computed via the standard Kalman method using $A_{c}$ and $C_{c}$. A loop transfer recover (LTR) design can be implemented by varying the state covariance matrix according to Eq. (31) 


\section{Nominal Flutter Control}

The nominal flutter controller consists of an optimal regulator with state feedback. The LQR state feedback gains are computed using $A_{w}^{f}$, which includes the stable closed loop tracking feedback controller defined above, and $B_{w}^{f}$, which corresponds to the control surfaces allocated for flutter suppression. This system is described as:

$$
\dot{x}_{w}=\underbrace{\left[A_{w}^{r b}+B_{w}^{r b} K_{x}^{r b}\right]}_{A_{w}^{f}} x_{w}+\underbrace{\left[\begin{array}{c}
0 \\
B_{c}^{f}
\end{array}\right]}_{B_{w}^{f}} u^{f}+\underbrace{\left[\begin{array}{c}
-1 \\
0
\end{array}\right]}_{B_{r}} \gamma_{c} .
$$

This controller is a regulator that attempts to drive the structural states of the plant to zero. Thus, LQR state weights for $Q_{f}$ are typically set to be non-zero only at those diagonal positions. The resulting nominal optimal flutter control law takes the form

$$
u_{n o m}^{f}=K_{n o m}^{f} \underbrace{\left[\begin{array}{c}
x_{i n t} \\
x_{r b} \\
\hat{x}_{e}
\end{array}\right]}_{\hat{x}},
$$

where, again, $\hat{\bar{x}}$ includes perfect knowledge of the rigid body and integrated error state are assumed, but all other states are estimated from the observer.

\section{E. Adaptive Flutter Control}

The nominal flutter controller is augmented with an adaptive portion according to

$$
u^{f}=\underbrace{K_{\text {nom }}^{f} \hat{\bar{x}}}_{u_{\text {nom }}^{f}}+\underbrace{K_{x}(t) \hat{\bar{x}}+K_{y}(t)(y-\hat{y})}_{u_{a d}^{f}} .
$$

The above nominal LQG flutter controller is designed as a regulator that attempts to drive the weighted states (according to $Q_{f}$ ) to zero. However, since $x_{i n t}$ and $x_{r b}$ are included in feedback, a reference model where the states are not assumed to be zero is utilized. Thus, the original MRAC OCM adaptive laws are used such that $e=x_{r e f}-\hat{\bar{x}}$. $K_{x}(t)$ and $K_{y}(t)$ adapting according to

$$
\begin{aligned}
& \dot{K}_{x}=B_{w}^{f^{T}}\left(P e+\nu_{x} A_{r e f}^{-T} P B_{w}^{f} K_{x} \hat{\bar{x}}\right) \hat{\bar{x}}^{T} \Gamma_{x} \\
& \dot{K}_{y}=B_{w}^{f^{T}}\left(P e+\nu_{y} A_{r e f}^{-T} P B_{w}^{f} K_{y}(y-\hat{y})\right)(y-\hat{y})^{T} \Gamma_{x} .
\end{aligned}
$$

Here $A_{\text {ref }}$ is the ideal Hurwitz reference model defined by $A_{\text {ref }}=A_{w}^{f}+B_{w}^{f} K_{n o m}^{f}$, and $P=P^{T}$ is the solution to the standard Lyapunov equation based on the reference model $P A_{r e f}+A_{r e f}^{T} P=-Q$ with $Q=Q^{T}>0$. In the next section, a simulation of this system is presented.

\section{Results}

\section{A. Nominal Control}

In the first scenario, the trim condition is set to just below the flutter boundary with an altitude of $25,000 \mathrm{ft}$ at Mach 0.78. However, the true plant model is above the flutter boundary at the same altitude, but with a speed of Mach 0.80. Refer to Fig. 11. Also, a linear plant perturbation is applied such that $\delta_{A}=0.005$, and $\delta_{B}=-0.005$. If nominal (non-adaptive) LQG tracking control is used to track a simple flight path angle doublet without flutter suppression $\left(u=u_{l q g}^{r b}\right)$, the resulting closed loop system contains two unstable poles at $0.1848 \pm 39.69$ and goes unstable due to flutter as shown in Fig. 12.

Next, the same scenario is run, but with non-adaptive flutter control included so that $u=u_{l q g}^{r b}+u_{n o m}^{f}$ with $u_{\text {nom }}^{f}$ defined in Eq. (45). Loop transfer recovery is applied with $\rho=0.5$. This produces a stable closed loop system, but the trim point difference and the additional $A$ and $B$ matrix perturbation results in high frequency oscillations in all control surface inputs and vertical accelerometer outputs. See Fig. 13. Note that the oscillations get worse as the flutter is excited from tracking the flight path angle. 

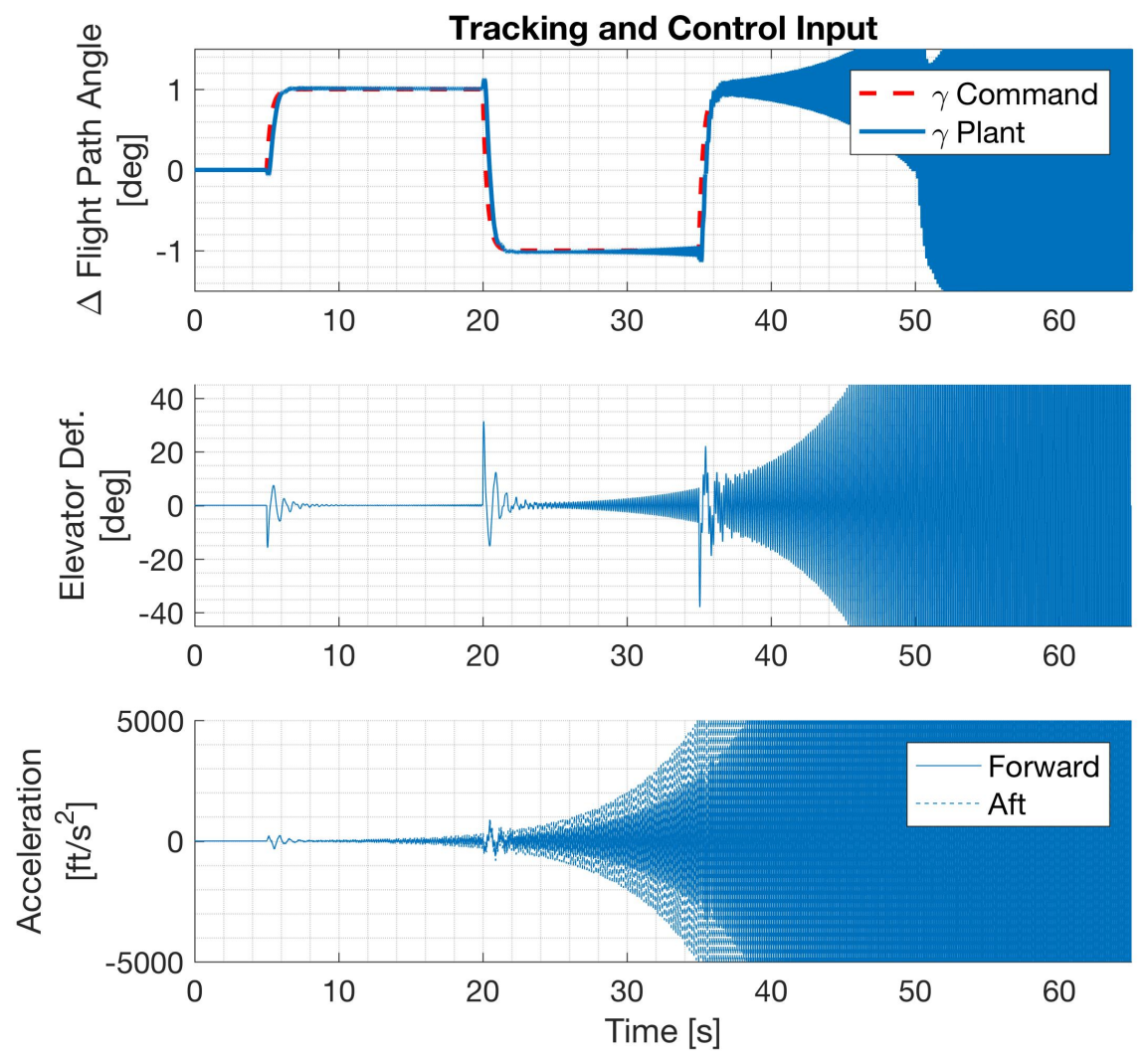

Figure 12. Nominal tracking control without flutter control. Control/plant model trim point separation across the flutter boundary with additional minor perturbation.

\section{B. Adaptive Flutter Suppression}

It is desired to augment the above system with adaptive MRAC OCM control in order to improve the performance. First, a reference model is created that is stable and tracks well. Recall that $A_{r e f}=A_{w}^{f}+$ $B_{w}^{f} K_{\text {nom. }}^{f}$. Selecting a trim point of $25,000 \mathrm{ft}$ and Mach 0.70 to calculate $A_{\text {ref }}$ results in a Hurwitz matrix that tracks well since it is significantly below the flutter boundary.

Next, the asymptotic stability property of OCM is used to find stable regions for the adaptive controller. This simulation is simplified from the above analysis by utilizing only the adaptive $K_{x}(t)$ term. Thus, the flutter control law is

$$
u^{f}=K_{\text {nom }}^{f} \hat{\bar{x}}+K_{x}(t) \hat{\bar{x}}
$$

where $K_{x}(t)$ adapts according to (47).

The fist step in tuning the OCM controller is to find the stable regions for $\nu_{x}$ similar to what was done in the above toy problems. In this case, however, there is a slight difference due to the structure of the learning rate gain $\Gamma_{x}$. The asymptotic closed loop matrix $A_{c l}^{\infty}$ from Eq. (18) is evaluated with $K_{a d}^{\infty}$ defined as

$$
K_{a d}^{\infty}=-\left(B_{c}^{T} A_{r e f}^{-T} P B_{c}\right)^{-1} B_{c}^{T} P G_{x} \frac{1}{\nu_{x}}
$$

where $G_{x}$ is a matrix with dimensions of $\Gamma_{x}$ consisting of zeros where $\Gamma_{x}$ is zero, and ones where it is non-zero. Since the portions of $K_{x}$ related to the other states are not adapting from the zero initial condition, they are filtered out of the asymptotic analysis using the $G_{x}$ term. It is found that good performance is achieved when using non-zero $\Gamma_{x}$ diagonal entries at the $x_{i n t}$ state and the elastic position states. $A_{c l}^{\infty}$ is then found to be stable for values of $\nu_{x}$ greater than 32 .

The diagonal entry of $\Gamma_{x}$ corresponding to the $x_{i n t}$ state is set to 0.00035 , and those corresponding to the elastic position states are set to 0.0021. $\nu_{x}$ is set to 35 . Comparing the adaptive controller performance 

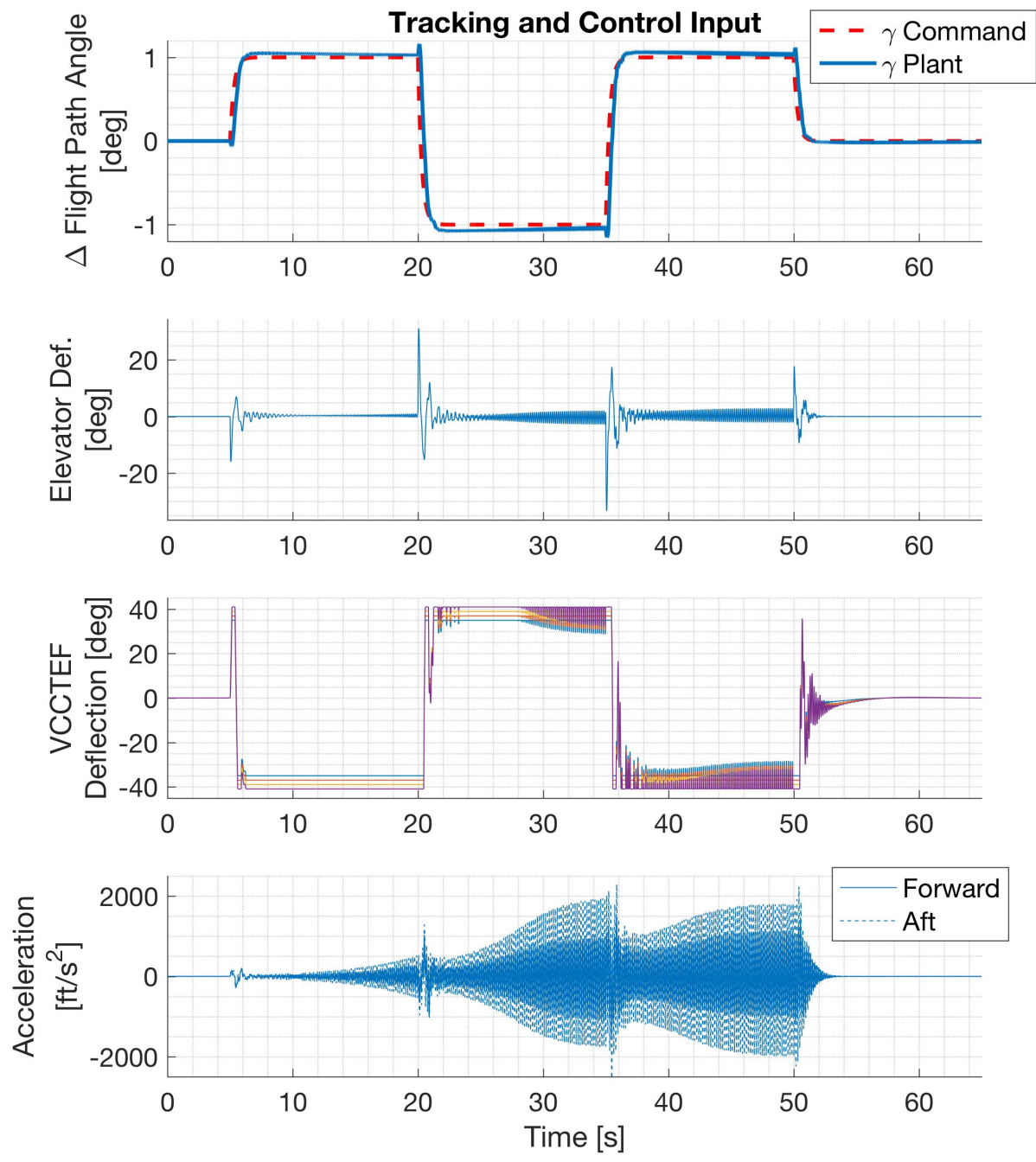

Figure 13. Nominal tracking and flutter control with control/plant model trim point separation across the flutter boundary with additional minor perturbation.

of Fig. 14 to that of Fig. 13, the magnitude and oscillations of the accelerometer outputs is significantly reduced. The smoother response of the plant with adaptive control also significantly reduces the high frequency oscillations on the elevator input despite the flutter controller not having command of the elevator. While the VCCTEF flaps still exhibit some high frequency oscillation, it has been reduced from the nominal control results, and more notably, the oscillations diminish with time as the adaptive gains evolve. The last plot in Fig. 14 shows the progression of the adaptive gains. Note that they remain bounded during tracking maneuvers, and that they converge during the last 15 seconds of level flight. This is consistent with what was predicted for steady state stability using the asymptotic analysis of $A_{c l}^{\infty}$. 

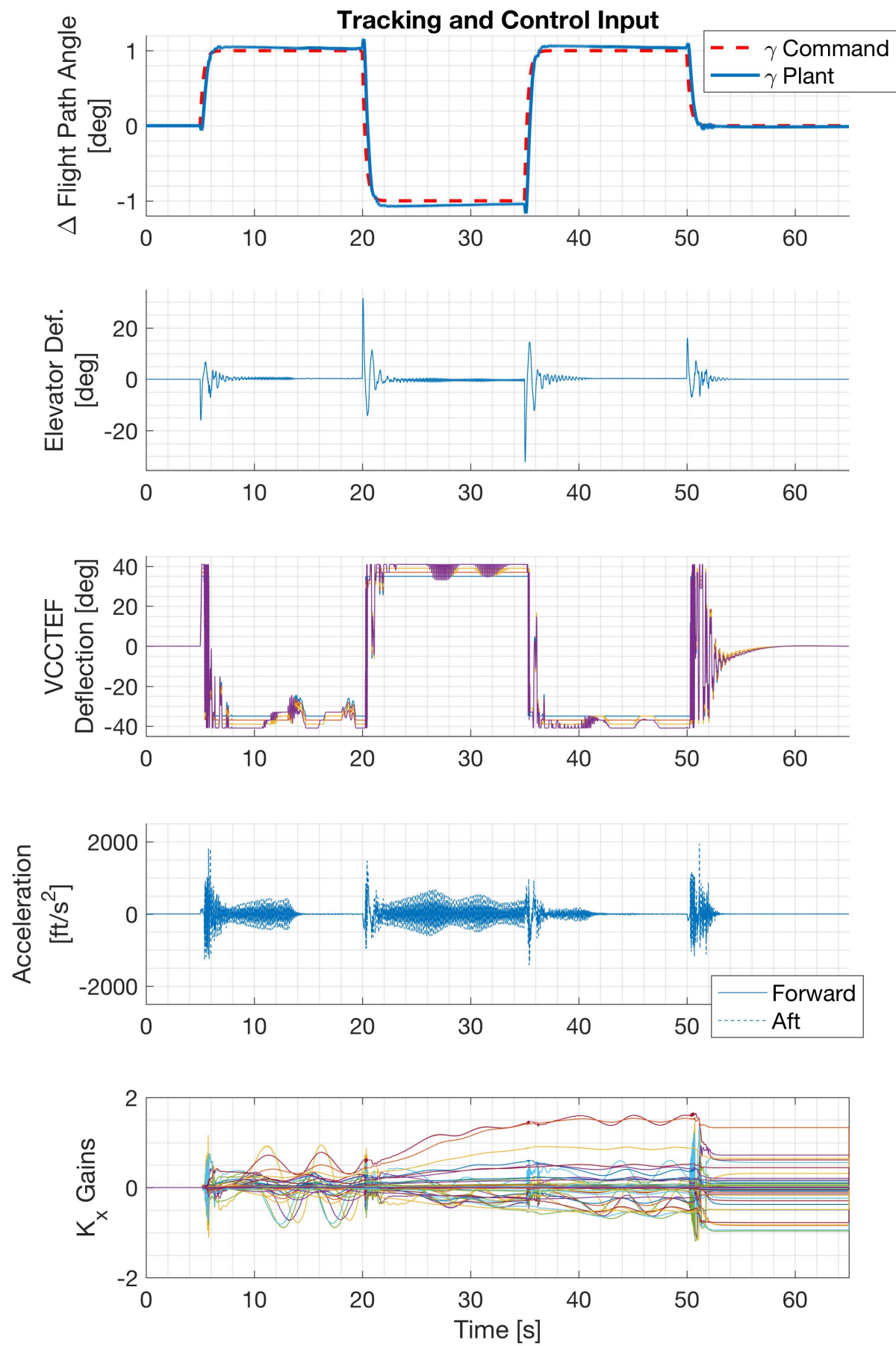

Figure 14. Tracking and adaptive flutter control with control/plant model trim point separation across the flutter boundary with additional minor perturbation.

\section{Conclusions}

The OCM controller has already demonstrated that it can provide measurable robustness to adaptive MRAC control via the additional tuning parameter $\nu$. In this paper, the bounds of this parameter are explored in relation to several plant and controller attributes that affect the amount of stability and ro- 
bustness available to the adaptive controller. Two small example models are used to show that time delay margin robustness can be accurately predicted relative to $\nu$, and that stable bounds for $\nu$ given linear plant perturbation and controller design can be easily computed using OCM's asymptotic properties. While plant perturbations negatively affect robustness, causing the stable regions of $\nu$ to shrink, baseline controllers tuned for increased robustness cause them to grow. Finally, the asymptotic property of the OCM controller was utilized to aid in the tuning and implementation of a large scale (63 states) OCM augmented flutter suppression controller applied to the GTM ASE model. While the asymptotic property is very useful for evaluating steady state stability boundaries of the OCM controller, future work can be done to better understand how the $\nu$ tuning parameter quantitatively affects the transient regions of adaptive control.

\section{References}

${ }^{1}$ Rohrs, C., Valavani, L., Athans, M., and Stein, G., "Robustness of continuous-time adaptive control algorithms in the presence of unmodeled dynamics," IEEE Transactions on Automatic Control, Vol. 30, No. 9, 1985, pp. 881-889.

${ }^{2}$ Cao, C. and Hovakimyan, N., "Design and Analysis of a Novel Adaptive Control Architecture With Guaranteed Transient Performance," IEEE Transactions on Automatic Control, Vol. 53, No. 2, 2008, pp. 586-591.

${ }^{3}$ Calise, A. J. and Yucelen, T., "Adaptive loop transfer recovery," Journal of Guidance, Control, and Dynamics, Vol. 35, No. 3, 2012, pp. 807-815.

${ }^{4}$ Yucelen, T. and Calise, A., "A Kalman filter optimization approach to direct adaptive control," AIAA Guidance, Navigation, and Control Conference, 2009, p. 5858.

${ }^{5}$ Ioannou, P. A. and Kokotovic, P. V., "Paper: Instability Analysis and Improvement of Robustness of Adaptive Control," Automatica, Vol. 20, No. 5, Sept. 1984, pp. 583-594.

${ }^{6}$ Narendra, K. and Annaswamy, A., "A new adaptive law for robust adaptation without persistent excitation," IEEE Transactions on Automatic control, Vol. 32, No. 2, 1987, pp. 134-145.

${ }^{7}$ Nguyen, N., Krishnakumar, K., and Boskovic, J., "An optimal control modification to model-reference adaptive control for fast adaptation," AIAA Guidance, Navigation and Control Conference and Exhibit, 2008, p. 7283.

${ }^{8}$ Nguyen, N. T., "Optimal control modification for robust adaptive control with large adaptive gain," Systems \& Control Letters, Vol. 61, No. 4, 2012, pp. 485-494.

${ }^{9}$ Nguyen, N. T., "Adaptive Control for linear uncertain systems with unmodeled dynamics revisited via optimal control modification," AIAA Guidance, Navigation, and Control (GNC) Conference, 2013, p. 4988.

${ }^{10}$ Nguyen, N., Hanson, C., Burken, J., and Schaefer, J., "Normalized Optimal Control Modification and Flight Experiments on NASA F/A-18 Aircraft," Journal of Guidance, Control, and Dynamics, Vol. 40, No. 4, 2017/05/31 2016, pp. 1061-1075.

${ }^{11}$ Nguyen, N. T. and Swei, S. S.-M., "Adaptive Linear Quadratic Gaussian Optimal Control Modification for Flutter Suppression of Adaptive Wing," AIAA Infotech@ Aerospace, 2015, p. 0118.

${ }^{12}$ Al-Jiboory, A. K., Zhu, G., Swei, S. S.-M., Su, W., and Nguyen, N. T., "LPV modeling of a flexible wing aircraft using modal alignment and adaptive gridding methods," Aerospace Science and Technology, Vol. 66, 2017, pp. 92-102.

${ }^{13}$ Doyle, J., "Guaranteed margins for LQG regulators," IEEE Transactions on automatic Control, Vol. 23, No. 4, 1978, pp. $756-757$.

${ }^{14}$ Athans, M., "A tutorial on the LQG/LTR method," American Control Conference, 1986, IEEE, 1986, pp. $1289-1296$.

${ }^{15}$ Lavretsky, E., "Adaptive output feedback design using asymptotic properties of lqg/ltr controllers," IEEE Transactions on Automatic Control, Vol. 57, No. 6, 2012, pp. 1587-1591.

${ }^{16}$ Nguyen, N., Trinh, K., Reynolds, K., Kless, J., Aftosmis, M., and Urnes, J., "Elastically shaped wing optimization and aircraft concept for improved cruise efficiency," 51st AIAA Aerospace Sciences Meeting Including the New Horizons Forum and Aerospace Exposition, 2013, p. 141.

${ }^{17}$ Nguyen, N. T., Ting, E., Chaparro, D., Drew, M. C., and Swei, S. S.-M., "Multi-Objective Flight Control for Drag Minimization and Load Alleviation of High-Aspect Ratio Flexible Wing Aircraft," 58th AIAA/ASCE/AHS/ASC Structures, Structural Dynamics, and Materials Conference, 2017, p. 1589.

${ }^{18}$ Nguyen, N. T., Drew, M. C., and Swei, S. S.-M., "Adaptive Modal Identification for Flutter Suppression Control," AIAA Infotech@Aerospace, 2016, p. 0400. 